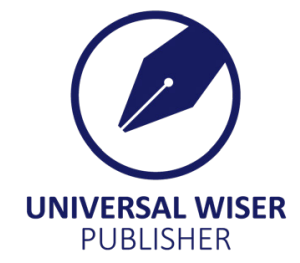

\title{
Congestion Management in Deregulated Electricity Market with Generator Sensitivity Effects
}

\author{
Ali Ghasemi-Marzbali \\ Department of Computer and Electrical Engineering, Mazandaran University of Science and Technology, Babol, Iran \\ Email: ali.ghasemi@ustmb.ac.ir
}

\begin{abstract}
This paper proposes a novel modified virus colony search (MVCS) algorithm to solve congestion management (CM) problem in the deregulated electricity market considering generator sensitivity and rescheduling of active powers. Thus, the generator output has been recognized by generator sensitivity index to manage transmission line limitation so as to get maximum efficiency in power transmission. The CM model is formulated based on a single objective function to minimize final cost by optimal planning of active powers responding to generators. To develop the exploration and exploitation of VCS, proposes some modifications into local and global parts which they make the best stability in VCS structure. To reveal the MVCS performance, it is assessed on three well-known power systems, IEEE 30-bus and IEEE 118-bus, which the numerical results show acceptable performance compare to other available methods.
\end{abstract}

Keywords: Congestion management, Optimization, Electricity market, Virus search colony

\section{Introduction}

Power system during the recent years is moved from vertically and monopolistic model to restructured, independent and competitive model. In the vertical structure, the government has a monopoly role in controlling all sections e.g., distribution companies (DisCos), transmission companies (TransCos) and generation companies (GenCos) ${ }^{[1]}$. In the restructured model, the bilateral contracts between the power market participants under the Independent System Operator (ISO) managing raise new engineering fields in operation and planning ${ }^{[2]}$. According to the deregulation mechanism, this can be deduced that the ultimate goal is efficiency and social welfare increases in the programmed value based on the current and installed power system capacity. Also, the restructured power system prepares many ancillary services, wherein, congestion management is one of important service to control the transmission line and to contain the bilateral transactions ${ }^{[3]}$. Since the power system operator tries to transmit more power for consumers at the least planning cost, a result of a high-density user crossing point is that it makes a restricted access that bans the supply of energy to customers at an expensive cost ${ }^{[4]}$. This means that these customers should pay more for their requested power than they have if there was sufficient transfer capacity for the transfer of economic transactions. According to the review-knowledge, there are several congestion management methods which they try to present a new method solving these difficulties in a transmission line. In [5] proposed a market-based system to improve distribution system congestions throughout a centralized synchronized home energy management scheme. The power system operator employs dynamic and daily power based on distribution tariffs to handle congestions induced. In [6] suggested teaching-learning-based optimization algorithm for CM problem in the restructured electricity market. They consider security limitations such as load bus voltage and line loading. In [7] employed the capability of distributed generation to reduce the transmission line congestion in a power system. To reduce congestion, optimal power flow and accessible transfer ability-based approaches for giving out thyristor controlled series compensator by means of the congestion rent contribution method based on location marginal price is proposed in [8]. Also, the gravitational search algorithm is used to control congestion in a restructured electricity market. In [9] employed particle swarm optimization with time-varying acceleration coefficients to solved optimal congestion management problem in a restructured electricity market. To the best review-knowledge of published papers based on congestion management problem with ancillary devices and optimal managing, there is yet a gap for more a powerful framework. Motivated by this note, the main idea of this paper is offering a useful and practical framework of congestion management in the restructured power system. In addition, to cover the previous optimization algorithm shortages, an improved algorithm based on 
virus colony search is proposed ${ }^{[10]}$. The proposed method is a new population-based method which has faster and better performance compare to other optimization methods. Therefore, the main contributions are:

i) Modeling of a practical model of congestion management problem

ii) Consider generator sensitivity and optimal power flow simultaneously

iii) Propose a modified VCS algorithm based on local and global searches

iv) Evaluate the proposed model with three two well-known test systems.

\section{Congestion Management Formulation}

The objective is investigating of proposed VCS algorithm with generator sensitivity to manage congestion and congestion re-dispatch so as to minimize the final $\operatorname{cost}^{\left[{ }^{[9]}\right.}$. Hereby, it can express as follows:

$$
\operatorname{Min} \sum_{g=1}^{N g} I c_{g}\left(\Delta P_{g}\right) \Delta P_{g}
$$

Subject to:

$$
\begin{aligned}
& \sum_{g=1}^{N g}\left(G S_{g}^{i j} . \Delta P_{g}\right)+F_{l}^{0} \leq F_{l}^{\max } \\
& \Delta P_{g}^{\min } \leq \Delta P_{g} \leq \Delta P_{g}^{\max } ; g=1,2, \ldots, N g \\
& \Delta P_{g}^{\min }=P_{g}^{\min }-P_{g} ; \Delta P_{g}^{\min }=P_{g}^{\min }-P_{g}
\end{aligned}
$$

where, $\mathrm{IC}_{\mathrm{g}}$ is the incremental (decremental) cost of generator $\mathrm{g}$. $\mathrm{N}_{\mathrm{g}}$ and $\Delta \mathrm{P}_{\mathrm{g}}$ denote the number of participating generators and active power adjustment at bus g, respectively. $\Delta \mathrm{P}_{\text {min }}^{\mathrm{g}}$ and $\Delta \mathrm{P}_{\text {max }}^{\mathrm{g}}$ refer to the minimum and maximum boundary values of generator $\mathrm{g}$. Furthermore, $\mathrm{P}_{\text {min }}^{\mathrm{g}}$ and $\mathrm{P}_{\text {max }}^{\mathrm{g}}$ dictate the minimum and maximum limitation to $\mathrm{g}^{\text {th }}$ generator. The goal is finding GS value of $\mathrm{g}^{\text {th }}$ generator on the interconnected line between buses $i$ and $j$, which it can change as well as active power flow. It can be calculated by:

$$
G S_{g}^{i j}=\frac{\Delta P_{i j}}{\Delta P_{G g}}=\frac{\partial P_{i j}}{\partial \theta_{i}} \cdot \frac{\partial \theta_{i}}{\partial P_{G g}}+\frac{\partial P_{j i}}{\partial \theta_{j}} \cdot \frac{\partial \theta_{j}}{\partial P_{G g}}
$$

where, $\theta_{\mathrm{i}}$ and $\theta_{\mathrm{j}}$ denotes the phase angle at buses $\mathrm{i}$ and $\mathrm{j} . \Delta \mathrm{P}_{\mathrm{ij}}$ is active power change between buses $\mathrm{i}$ and $\mathrm{j}$. and $\Delta \mathrm{P}_{\mathrm{Gg}}$ is changed value in active power of generator $\mathrm{g}$. The power flow can be got by:

$$
P_{i j}=-V_{i}^{2} \cdot G_{i j}+V_{i} V_{j} \cdot G_{i j} \cdot \cos \left(\theta_{i}-\theta_{j}\right)+V_{i} V_{j} \cdot B_{i j} \cdot \sin \left(\theta_{j}-\theta_{i}\right)
$$

\section{Improved Virus Colony Search}

\subsection{Standard VCS}

The VCS is one method based on the population of viruses and host cells, founded on two behaviors of infecting the host cell and the dispersion or reproduction of the virus. Since the proposed developed model requires its mathematical basis, its mathematical formulation is fully described in this section. Refer to reference ${ }^{[10]}$ for further study. For reader convenience and a short introduction of the standard VCS formulations, Fig 1 shows its procedures.

\subsection{Modified VCS Algorithm}

The most important disadvantage of the standard VCS can be found in its rapid convergence. In this study, the following modifications are proposed. Based on VCS:

$$
V_{p o p_{i}^{\prime}}=\operatorname{Gaussian}\left(G_{\text {best }}^{g}, \tau\right)+\left(r_{1} \cdot G_{\text {best }}^{g}-r_{2} V_{\text {pop }}\right)
$$


where $r_{1}$ and $r_{2}$ are two random parameters. Hereby they create weaknesses in searching process. To develop its performance, the following time-varying formulation is suggested:

$$
V_{\text {pop }}=\operatorname{Gaussian}\left(G_{\text {best }}^{g}, \tau\right)+\left(\rho^{L} \beta_{t, i}^{L}, G_{\text {best }}^{g}-\rho^{g} \beta_{t, i}^{g} V_{p o p_{i}}\right)
$$

\section{Start}

01 Set initial parameters such as population size $(N)$, maximum iteration,

$$
\left(\text { Iter }_{\max }\right), \lambda=N / 2
$$

02 Generate random-based population $\left(V_{\text {pop }}\right)$

03 Calculate fitness value for each solution

04 while iter $^{<}$Iter $_{\max }$

05 for $\mathrm{i}=1: \mathrm{N}$ /Viruses diffusion/

06

$$
V_{p o p_{i}^{\prime}}=\operatorname{Gaussian}\left(G_{\text {best }}^{g}, \tau\right)+\left(r_{1} \cdot G_{\text {best }}^{g}-r_{2} V_{p o p_{i}}\right)
$$

07 end

08 Check boundary conditions

09 Calculate $V_{\text {pop }}$ ' fitness values and update $V_{\text {pop }}$

10 for $\mathrm{i}=1: \mathrm{N}$ /Host cells infection/

$11 \quad H_{\text {pop }}^{g}=X_{\text {mean }}^{g}+\sigma_{i}^{g} \times N_{i}\left(0, C^{g}\right)$

12 end

13 Check boundary conditions

14 Calculate $\mathrm{H}_{\text {pop }}$ fitness values and update $\mathrm{V}_{\text {pop }}$

15 Set the best $\lambda$ by $X_{\text {mean }}^{g+1}=\gamma^{-1} \sum_{i=1}^{\gamma} \omega_{i} V_{\text {popp }}^{\text {best }} \mid \omega_{i}=\ln (\gamma+1) / \sum_{j=1}^{\gamma}(\ln (\gamma+1)-\ln (j))$

16 Compute $\mathrm{P}_{\mathrm{r}}$ by $\mathrm{P}_{\text {rrank }}(\mathrm{i})=(\mathrm{N}-\mathrm{i}+1) / \mathrm{N}$

17 for $\mathrm{i}=1: \mathrm{N}$

18 for $\mathrm{j}=1: \mathrm{d}$

19 if $\mathrm{r}>\mathrm{P}_{\mathrm{ri}}$

$20 \quad \mathrm{~V}_{\mathrm{popi}, j^{\prime \prime}}=\mathrm{V}_{\mathrm{popk}, \mathrm{j}^{-}}$rand $\left(\mathrm{V}_{\mathrm{poph}, \mathrm{j}^{-}}-\mathrm{V}_{\mathrm{popi}, \mathrm{j}}\right)$;

$21 \quad$ else

$22 \quad \mathrm{~V}_{\text {popi }, \mathrm{j}}{ }^{\prime \prime}=\mathrm{V}_{\text {popi }, \mathrm{j}}$

23 end

24 end

25 end

26 Calculate $\mathrm{V}_{\text {pop }}$ " fitness values and update $\mathrm{V}_{\text {pop }}$

27 end

Finish

Figure 1. Pseudo code for standard VCS, where, $\mathrm{v}_{\mathrm{pop}}$ is population size, $G_{g}^{\text {best }}$ is the best solution of the generation $g, \mathrm{~N} i(0, \mathrm{Cg})$ is a normal distribution with mean 0 and $D \times D$ covariance matrix $C g, g$ is the current generation, $D$ is the dimension of the problem and $\sigma g>0$ is the step size, $\omega_{i}$ is the recombination weight and $i$ denotes the index of $i^{\text {th }}$ best individuals.

$\beta_{t, i}^{L}=-(t-i)^{-\vartheta} \sum_{i=1}^{t-1}(t-i)^{\vartheta}$

$\beta_{t, i}^{g}=(t-i)^{-\vartheta} \sum_{i=1}^{t-1}(t-i)^{-\vartheta}$

where $\beta_{t, i}^{L}$ and $\beta_{t, i}^{g}$ deotes cognitive and social coefficients. $\rho^{L}$ and $\rho^{g}$ are two random variables in range $(0,1) . \vartheta$ and subscript $\mathrm{t}$ are positive value and iteration number, respectively. 


\section{Simulation Results}

The proposed method is evaluated on two well-known test systems, IEEE-30 bus and IEEE-118 bus. The obtained results with proposed MVCS compare to several version of particle swarm optimization, CPSO ${ }^{[9]}$, PSO-TVAC ${ }^{[9]}$ and PSO-TVIW ${ }^{[9]}$. Also, they have performed on PC with Intel (R) Core (TM) Duo CPU @ 2.53GHz, 4GB of RAM using MATLAB 2011a.

\subsection{IEEE 30-bus System}

At first test system, the IEEE 30-bus power system with six generating units and forty one lines is employed. Its configuration is shown in Fig. 2 and the system data can be found in [11]. This case, Bus 1 is considered as the reference bus or slack. A congested line between buses 1 and 2 exists as shown in Table 1. Table 2 present GS values of 6 generator units. Considering GS values, all generators are selected for re-dispatch. The obtained values for GS index in the IEEE 30bus system are large; therefore, it dictates power system to use all of them in re-dispatch program to relieve the congested line. In this way, generator having large GS value considered to avoid the computation time.

Table 1. A congested line on the IEEE 30-bus system

\begin{tabular}{cccc}
\hline Congested line & Active power flow (MW) & Line limit (MVA) & Overload (MW) \\
\hline 1 to 2 & 170 & 130 & 40 \\
\hline
\end{tabular}

Table 2. Generation sensitivity of 6 units on the IEEE 30-bus system

\begin{tabular}{ccccccc}
\hline Gen no & 1 & 3 & 5 & 8 & 11 & 13 \\
\hline GS 1_2 & 0 & -0.8908 & -0.8527 & -0.7394 & -0.7258 & -0.6869 \\
\hline
\end{tabular}

Fig 3 shows the graphical results for convergence, it can be obvious that the proposed MVCS has fast performance as well. The average active power correction and GS values for all generators is shown in Fig 4. Regarding 30 trial simulation, the obtained statistical results with MVCS and other optimization algorithms tabulated in Table 3. According to this table, the proposed algorithm obtains the minimum re-dispatch cost solution of \$ 212.4, whereas PSO-TVAC, CPSO and PSOTVIW obtained $\$ 237.9 / \mathrm{h}, \$ 240.3 / \mathrm{h}$ and $\$ 239.2 / \mathrm{h}$, respectively. In addition, the proposed MVCS find this solution with lowest standard deviation 1.02, whereas PSO-TVAC, CPSO and PSO-TVIW supply 1.6, 48.2 and 3.8, respectively ${ }^{[9]}$. To have a fair judgment, employed mismatch balance power index to compare the aforementioned algorithms:

$$
\text { mismatch power }=\sum_{i=1}^{N_{g}} \Delta P_{i}\left(G_{i}\right)=0
$$

The proposed index shows algorithm ability in solving complex mathematical problems and finding optimal solutions.

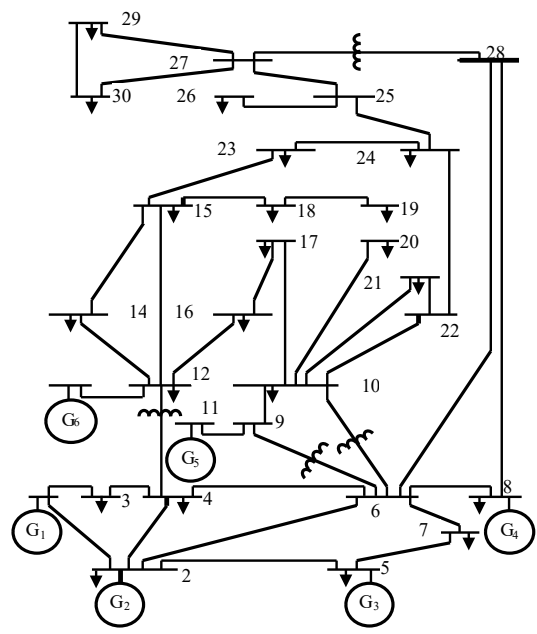

Figure 2. The IEEE 30-bus system configuration 


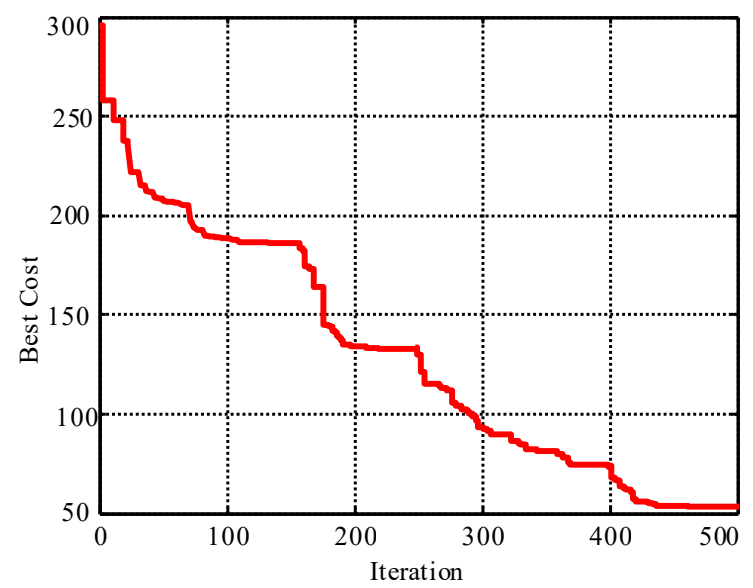

Figure 3. Evolution rate comparison CM problem in IEEE 30-bus test system

Table 3. Comparison of MVCS solutions on the IEEE 30-bus system

\begin{tabular}{ccccccccccc}
\hline Method & MW & $\Delta \mathrm{P} 1$ & $\Delta \mathrm{P} 2$ & $\Delta \mathrm{P} 5$ & $\Delta \mathrm{P} 8$ & $\Delta \mathrm{P} 11$ & $\Delta \mathrm{P} 13$ & $\begin{array}{c}\text { Total } \\
\Delta \mathrm{P}\end{array}$ & $\begin{array}{c}\text { Cost }(\$ \mathrm{~h}) \\
/ \mathrm{M})\end{array}$ & $\begin{array}{c}\text { Mismatch } \\
\text { power }\end{array}$ \\
\hline CPSO & Max & -66.1 & 28.9 & 23.3 & 18.1 & 6.2 & 3.7 & 146.3 & 403.1 & 14.10 \\
& Min & -47.9 & 18.6 & 16.5 & 11.3 & 2.8 & 0.1 & 97.2 & 240.3 & 1.40 \\
& Mean & -55.9 & 22.6 & 16.2 & 10.5 & 5.6 & 2.6 & 113.2 & 287.1 & 1.60 \\
PSO- & SD & 8.3 & 7.6 & 3.5 & 3.3 & 3.2 & 3.3 & 15.9 & 48.2 & ----- \\
TVIW & Max & -58.5 & 16.7 & 13.0 & 11.8 & 8.6 & 5.7 & 114.2 & 288.0 & -2.70 \\
& Min & -47.3 & 20.1 & 14.5 & 10.5 & 4.8 & 0.5 & 97.7 & 239.2 & 3.10 \\
& Mean & -50.1 & 18.9 & 13.2 & 9.2 & 5.9 & 4.1 & 101.4 & 253.1 & 1.20 \\
PSO- & SD & 2.8 & 3.5 & 5.4 & 3.3 & 3.5 & 6.1 & 13.3 & 3.8 & ---- \\
TVAC & Max & -51.1 & 22.0 & 14.7 & 8.8 & 6.2 & 1.0 & 103.8 & 254.9 & 1.60 \\
& Min & -47.3 & 25.1 & 16.0 & 7.6 & 0.6 & 0.0 & 96.7 & 237.9 & 2.00 \\
& Mean & -49.3 & 17.5 & 14.0 & 9.9 & 6.8 & 3.0 & 100.5 & 247.5 & 1.90 \\
MVCS & SD & 0.8 & 2.1 & 2.1 & 2.2 & 2.3 & 2.4 & 4.6 & 1.6 & ----- \\
& Max & -45.6 & 20.5 & 17.1 & 8.56 & 3.6 & 1.02 & 96.4 & 243.2 & 0.75 \\
& Min & -43.5 & 20.2 & 15.0 & 6.13 & 0.6 & 0.02 & 85.5 & 212.4 & 0.14 \\
& Mean & -45.4 & 22.3 & 15.1 & 6.56 & 0.9 & 1.07 & 91.3 & 231.1 & 0.52
\end{tabular}

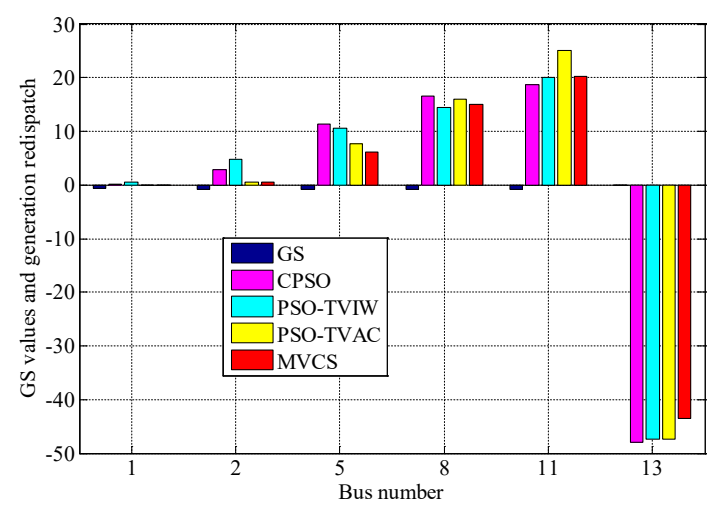

Figure 4. GS values and generation re-dispatch on the IEEE 30-bus system

\subsection{IEEE 118-bus System}

The configuration of the IEEE 118-bus system is shown in Fig 5 and data is available in [12]. Similar to the previous test system, slack bus is Bus 1. The congested line data is listed in Table 4. 
Table 4. A congested line on the IEEE 118-bus system

\begin{tabular}{cccc}
\hline Congested line & Active power flow (MW) & Line limit (MVA) & Overload (MW) \\
\hline 89 to 90 & 260 & 200 & 60 \\
\hline
\end{tabular}

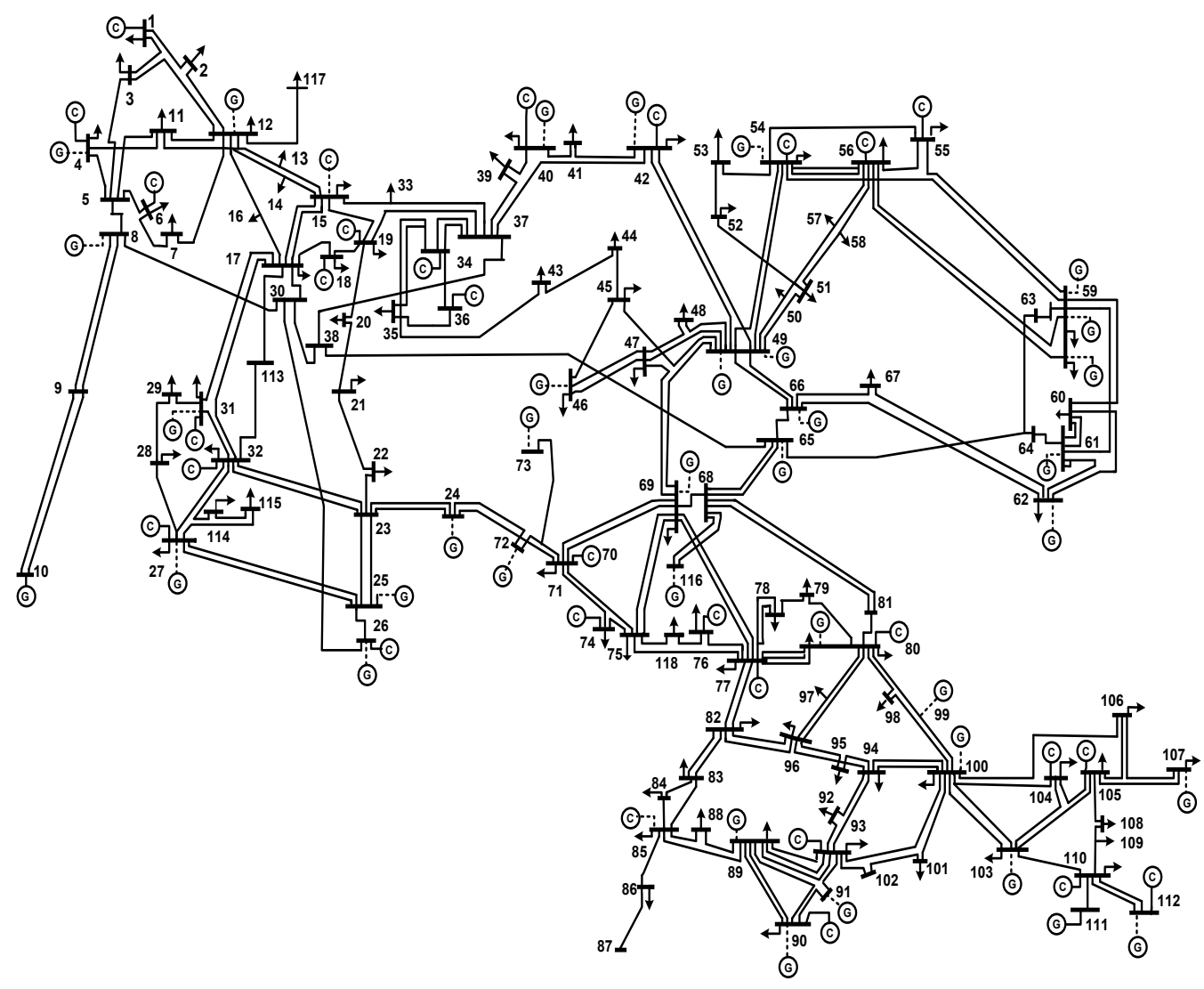

Figure 5. The IEEE 118-bus system configuration

The calculated GS is shown in Fig 6, the obtained results of GS values for all generator buses are compared in Table 5. This results shown, the generator buses $85,87,89,90$, and 91 have most considerable magnitude of GS. This implies that these generators could considerably affect on the congested line. Consequently, they are selected in re-dispatched progress.

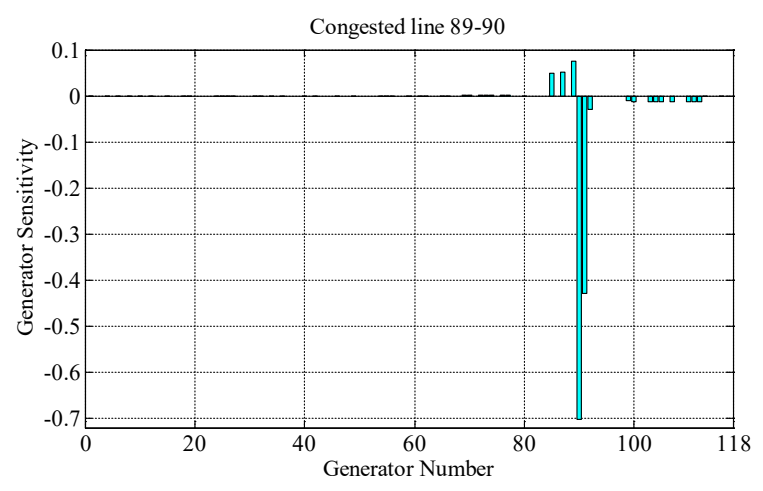

Figure 6. GS values of 54 units on the IEEE 118-bus system 
Table 5. GS values of 54 generators on the IEEE 118-bus system

\begin{tabular}{cccccc}
\hline Gen no. & $\begin{array}{c}\text { GS } \\
\left(10^{\wedge}-3\right)\end{array}$ & Gen no. & $\begin{array}{c}\text { GS } \\
\left(10^{\wedge}-3\right)\end{array}$ & Gen no. & $\begin{array}{c}\text { GS } \\
\left(10^{\wedge}-3\right)\end{array}$ \\
\hline 1 & 0 & 42 & -0.0375 & 80 & -0.9250 \\
4 & -0.0005 & 46 & -0.0242 & 85 & 50.068 \\
6 & -0.0001 & 49 & -0.0460 & 87 & 50.654 \\
8 & -0.0014 & 54 & -0.0838 & 89 & 74.455 \\
10 & -0.0014 & 55 & -0.0871 & 90 & -701.15 \\
12 & 0.0004 & 56 & -0.0854 & 91 & -427.90 \\
15 & 0.0021 & 59 & -0.1100 & 92 & -28.411 \\
18 & 0.0051 & 61 & -0.1160 & 99 & -9.391 \\
19 & 0.0046 & 62 & -0.1130 & 100 & -12.915 \\
24 & 0.1350 & 65 & -0.1350 & 103 & -12.737 \\
25 & 0.0484 & 66 & -0.0983 & 104 & -12.854 \\
26 & 0.0337 & 69 & 0.2120 & 105 & -12.772 \\
27 & 0.0451 & 70 & 0.3690 & 107 & -12.202 \\
31 & 0.0339 & 72 & 0.2326 & 110 & -12.274 \\
32 & 0.0477 & 73 & 0.3400 & 111 & -12.07 \\
34 & -0.0323 & 74 & 0.5410 & 112 & -11.747 \\
36 & -0.0329 & 76 & 0.8650 & 113 & 0.0110 \\
40 & -0.0343 & 77 & 0.0012 & 116 & -0.1750 \\
\hline
\end{tabular}

Regarding 30 trial simulation, the obtained solutions from MVCS algorithm and other available method ${ }^{[9]}$ are tabulated in Table 6. According to these results, MVCS algorithm gives the lowest re-dispatch cost of $\$ 822.4$, whereas PSO-TVAC found $829.5 / \mathrm{h}$ and CPSO and PSO-TVIW obtained $\$ \$ 819.7225 / \mathrm{h}$ and $\$ 853.8 / \mathrm{h}$, respectively. According to the mean and SD indices, MVCS algorithm make 22.3, whereas PSO-TVAC is 94, CPSO and PSO-TVIW provide 196.4 and 165.8 , respectively ${ }^{[9]}$.

The association connecting of GS values and power re-dispatch is shown in Fig 7. Since the buses 85, 87, and 89 have positive values, the installed generators in these buses should reduce their output. In return, the installed units at buses 90 and 91 make the negative GS values; therefore their generating output should be increased. Furthermore, the GS magnitude affects the amount of active power correction. In addition, Bus 1 employed to keep the power balance in a equilibrium point. Fig 8 shows the graphical results for convergence, it can be obvious that the proposed MVCS has fast performance as well.

Table 6. Comparison of MVCS solutions on the IEEE 118-bus system

\begin{tabular}{|c|c|c|c|c|c|c|c|c|c|c|}
\hline Method & MW & $\Delta \mathrm{P} 1$ & $\Delta \mathrm{P} 2$ & $\Delta \mathrm{P} 5$ & $\Delta \mathrm{P} 8$ & $\Delta \mathrm{P} 11$ & $\Delta \mathrm{P} 13$ & Total $\Delta \mathrm{P}$ & Cost $(\$ / h)$ & $\begin{array}{c}\text { Mismatch } \\
\text { power }\end{array}$ \\
\hline \multirow[t]{4}{*}{ CPSO } & $\operatorname{Max}$ & -5.1 & -6.4 & -8.6 & -122.9 & 117.8 & 18.9 & 279.8 & 1604.5 & -6.30 \\
\hline & Min & -5.1 & -27.3 & -27.5 & -28.9 & 68.1 & 25.9 & 182.7 & 875.0 & 5.20 \\
\hline & Mean & -5.9 & -15.3 & -31.5 & -62.0 & 85.1 & 26.8 & 226.6 & 1183.8 & -2.80 \\
\hline & SD & 4.4 & 8.4 & 11.4 & 17.5 & 23.2 & 14.6 & 30.5 & 196.4 & ----- \\
\hline \multirow[t]{4}{*}{ PSO-TVIW } & Max & -2.7 & -13.8 & -23.4 & -97.7 & 121.4 & 10.4 & 269.4 & 1497.8 & -5.80 \\
\hline & Min & -6.8 & -18.2 & -28.2 & -33.1 & 78.3 & 8.9 & 173.5 & 853.8 & 0.90 \\
\hline & Mean & -5.5 & -12.1 & -28.2 & -59.8 & 76.4 & 29.8 & 211.7 & 1088.4 & 0.60 \\
\hline & SD & 4.3 & 6.7 & 10.7 & 16.9 & 21.1 & 13.5 & 26.3 & 165.8 & ----- \\
\hline \multirow[t]{4}{*}{ PSO-TVAC } & Max & -5.9 & -6.2 & -6.5 & -96.2 & 80.1 & 30.5 & 225.5 & 1229.6 & -4.20 \\
\hline & Min & -0.8 & -12.1 & -13.9 & -52.3 & 81.6 & 3.3 & 163.8 & 829.5 & 5.80 \\
\hline & Mean & -4.4 & -10.3 & -22.0 & -58.5 & 69.4 & 24.7 & 189.3 & 970.7 & -1.10 \\
\hline & SD & 2.9 & 5.0 & 10.0 & 15.1 & 9.8 & 16.1 & 16.5 & 94.5 & ----- \\
\hline \multirow[t]{4}{*}{ MVCS } & Max & -4.8 & -7.4 & -6.8 & -76.4 & 80.2 & 26.7 & 202.3 & 1211.2 & 0.13 \\
\hline & Min & -1.1 & -11.1 & -14.1 & -50.5 & 86.3 & 3.5 & 166.6 & 822.4 & 0.52 \\
\hline & Mean & -2.2 & -12.2 & -25.2 & -52.3 & 70.1 & 13.1 & 175.1 & 965.13 & 1.04 \\
\hline & SD & 1.0 & 2.2 & 6.4 & 11.1 & 5.1 & 13.1 & 12.5 & 22.3 & ---- \\
\hline
\end{tabular}




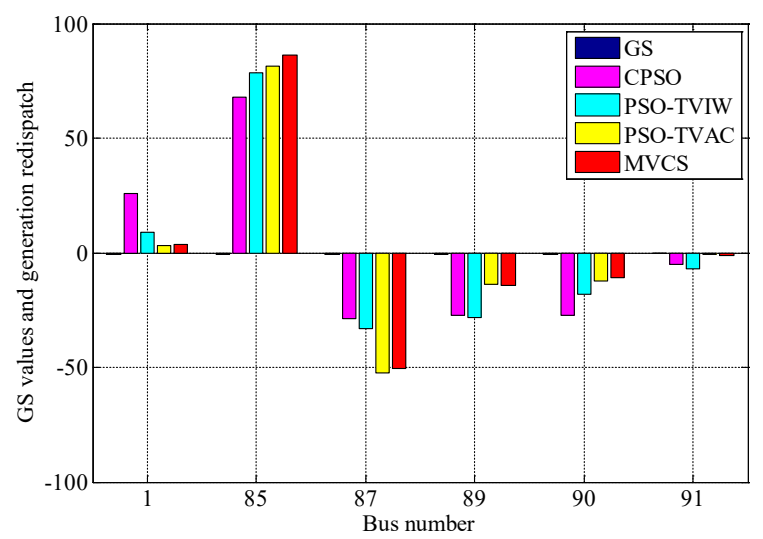

Figure 7. GS values and power re-dispatch on the IEEE 118-bus system

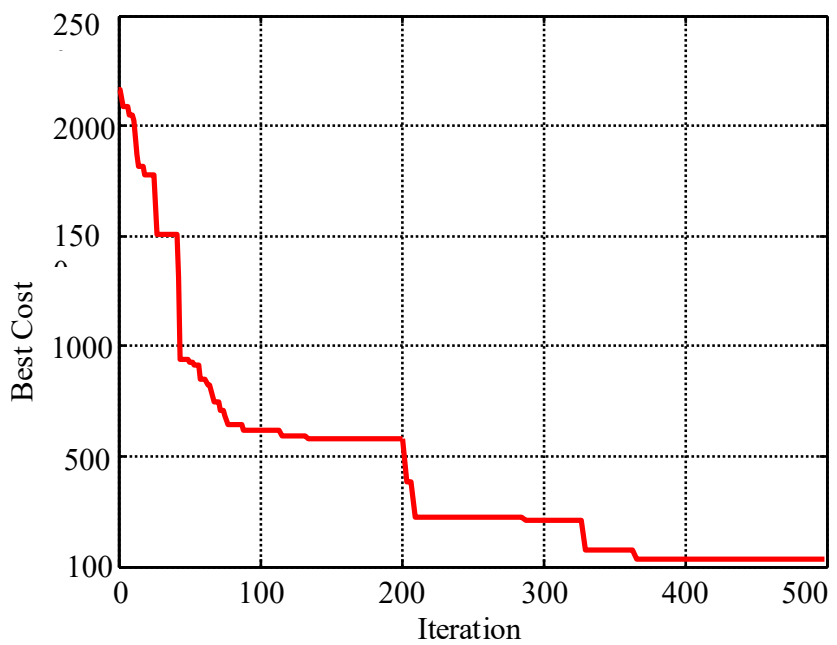

Figure 8. Evolution rate comparison CM problem in IEEE 118-bus test system

\subsection{Practical Indian 75-Bus System}

As a real test system, the Indian 75-bus system including 15 generator buses and 60 load buses is selected while its data is available in [13]. Bus 12 has been assigned as the Slack bus. When the base case power flows in various branches were computed, Line 71 was already overloaded. The unconstrained scheduled power flow of $401.65 \mathrm{MW}$ is recorded in line 71 , whose power flow limit is $400.00 \mathrm{MW}$. Hence, congestion has to be relieved by rescheduling active power generation of the participating generators. In this case, only 10 generators have shown strong influence on the congested line; thus, these have been selected for CM. The optimization algorithms are employed to optimally reschedule the active power of the selected generators for relieving congestion in line 71 . Table 7 gives the active power generation of the 10 participating generators before and after CM using MVCS, PSO ${ }^{[13]}$, FPSO ${ }^{[13]}$, and FDR-PSO ${ }^{[13]}$. 
Table 7. Active power generation before and after $\mathrm{cm}$ for the practical Indian 75-bus system

\begin{tabular}{|c|c|c|c|c|c|}
\hline Gen & Active Power & \multicolumn{4}{|c|}{ Active Power } \\
\hline \multirow[t]{4}{*}{ No. } & Generation (pu) & \multicolumn{4}{|c|}{ Generation (pu) } \\
\hline & before & \multicolumn{4}{|c|}{ after } \\
\hline & Congestion & \multicolumn{4}{|c|}{ Congestion Management } \\
\hline & Management & $\mathrm{PSO}^{[13]}$ & FPSO $^{[13]}$ & FDR-PSO $^{[13]}$ & MVCS \\
\hline 3 & 1.9248 & 1.7731 & 1.8000 & 1.9310 & 1.9983 \\
\hline 4 & 1.1653 & 0.9649 & 1.0000 & 0.9143 & 0.9823 \\
\hline 5 & 1.7572 & 1.9576 & 1.8000 & 1.9776 & 1.8220 \\
\hline 6 & 0.9680 & 1.0534 & 1.2000 & 1.0819 & 1.2113 \\
\hline 7 & 0.7005 & 0.5693 & 0.6000 & 0.9220 & 0.6119 \\
\hline 8 & 0.7469 & 0.9666 & 0.8000 & 0.8994 & 0.7726 \\
\hline 10 & 1.0237 & 0.7376 & 0.8000 & 0.6925 & 0.7387 \\
\hline 11 & 1.2258 & 1.2485 & 1.0900 & 1.0858 & 1.0222 \\
\hline 14 & 1.3312 & 1.3950 & 1.5000 & 1.2989 & 1.2635 \\
\hline 15 & 4.4229 & 4.5217 & 4.5400 & 4.4250 & 4.0198 \\
\hline
\end{tabular}

The optimization algorithms have been executed over five times on the studied test system to find out the robustness and effectiveness of the MVCS. Table 8 tabulated the best, worst, and mean values after CM for optimal rescheduling of the active powers of the participating generators. It can be seen in Table 8 that the MVCS algorithm obtained the minimum cost in relieving congestion.

Table 8. Comparison of $\mathrm{cm}$ methods for the practical Indian 75-bus system

\begin{tabular}{ccccc}
\hline & PSO $^{[18]}$ & FPSO $^{[18]}$ & FDR-PSO $^{[18]}$ & MVCS $^{[18}$ \\
\hline Best(Rs/MWh) & 5189.47 & 5075.44 & 5189.1 & 4985.91 \\
Worst(Rs/MWh) & 5243.81 & 5133.08 & 5213.77 & 5122.42 \\
Mean(Rs/MWh) & 5203.92 & 5098.34 & 5198.36 & 5071.02 \\
Time(Seconds) & 2.1207 & 2.4600 & 1.9573 & 1.89 \\
Losses(MW) & 207.8246 & 205.1068 & 206.6673 & 204.012 \\
Slack Bus Power(MW) & 1793.975 & 1788.776 & 1792.843 & 1782.213 \\
\hline
\end{tabular}

\subsection{Convergence Analyze}

To evaluate and compare different methods such as PSO, MVCS and standard VCS in converge term; the Langermann's benchmark is consider in this regard ${ }^{[14]}$. In other words, it is a nonlinear and multimodal optimization problem with two variables $\mathrm{x} 1$ and $\mathrm{x} 2$. It can be formulated by:

$$
\begin{aligned}
& f\left(x_{1}, x_{2}\right)=-\sum_{i=1}^{5} \frac{c_{i} \cos \left(\pi\left[\left(x_{1}-a_{i}\right)^{2}+\left(x_{2}-b_{i}\right)^{2}\right]\right)}{\frac{\left(x_{1}-a_{i}\right)^{2}+\left(x_{2}-b_{i}\right)^{2}}{\pi}} \\
& a=\left[\begin{array}{llllll}
3 & 5 & 2 & 1 & 7
\end{array}\right]^{T}, b=\left[\begin{array}{lllll}
5 & 2 & 1 & 4 & 9
\end{array}\right]^{T}, c=\left[\begin{array}{lllll}
1 & 2 & 5 & 2 & 3
\end{array}\right]^{T}
\end{aligned}
$$

The simulation results are listed in Table 9. It can be seen, the MVCS convergence is more faster compare to other optimization algorithms.

Table 9. Frequencies of convergence during of 100 trials

\begin{tabular}{ccccccc}
\hline Method & \multicolumn{5}{c}{ Range of Iteration } \\
& $0-50$ & $50-100$ & $100-200$ & $200-500$ & $500-1000$ & $>1000$ \\
\hline MVCS & 40 & 42 & 8 & 7 & 2 & 1 \\
VCS & 29 & 23 & 22 & 17 & 5 & 4 \\
PSO & 10 & 14 & 38 & 28 & 7 & 3 \\
\hline
\end{tabular}




\section{Conclusions}

In this paper, a novel modified virus colony search based on time-varying coefficient is suggested to solve the congestion management problem with generator sensitivity in a restructured electricity market. The proposed congestion management is formulated based on minimizing re-dispatch cost. The proposed model is evaluated with two well-known power systems under different operating conditions. MVCS algorithm has the advantage of being easy to implement without additional computational complexity. In addition, the convergence speed of this algorithm is acceptable. According to simulation results, the proposed algorithm can solve CM problem more efficiency compare to several version of PSO in the proposed power system.

\section{Conflict of interest}

The author declares no conflict of interest.

\section{References}

[1] Conejo A. J., Sioshansi R. (2018). Rethinking restructured electricity market design: Lessons learned and future needs. International Journal of Electrical Power \& Energy Systems, 98, 520-530.

[2] Brown D. P. (2018). Capacity payment mechanisms and investment incentives in restructured electricity markets. Energy Economics, 74, 131-142.

[3] Wang J., Pang X., Gao S., Zhao Y., Cui S. (2019). Assessment of automatic generation control performance of power generation units based on amplitude changes. International Journal of Electrical Power \& Energy Systems, 108, 1930.

[4] Yinliang X., Hongbin, S., Houde L., Qing F. (2018). Distributed solution to DC optimal power flow with congestion management. International Journal of Electrical Power \& Energy Systems, 95, 73-82.

[5] Mohammad Ali, F. G. et al. (2019). Congestion management in active distribution networks through demand response implementation. Sustainable Energy, Grids and Networks, 17, 1-13.

[6] Sumit V., Subhodip S., Mukherjee V. (2018). Optimal rescheduling of real power generation for congestion management using teaching-learning-based optimization algorithm. Journal of Electrical Systems and Information Technology, 5(3), 889-907.

[7] Rajagopal P., Vinod K. Y., Niranjan K. (2018). Flower pollination algorithm based multi-objective congestion management considering optimal capacities of distributed generations. Energy, 147, 980-994.

[8] Akanksha S.; Sanjay, K. J. (2019). Gravitational search assisted algorithm for TCSC placement for congestion control in deregulated power system. Electric Power Systems Research, 174, 105874.

[9] Panida B., Chanwit B., Weerakorn O. (2010). Optimal congestion management in an electricity market using particle swarm optimization with time-varying acceleration coefficients. Computers \& Mathematics with Applications, 60(4), 1068-1077.

[10] Li M.D., Zhao H., Wenig, X.W., Han T. (2016). A novel nature-inspired algorithm for optimization: Virus colony search. Advances in Engineering Software, 92, 65-88.

[11] Alsac O.; Stott B. (1974). Optimal load flow with steady-state security. IEEE Transactions on Power Apparatus and Systems, 93, 745-751.

[12] Devaraj D.; Yegnanarayana B. (2005). Genetic algorithm-based optimal power flow for security enhancement. IEE Proceedings - Generation, Transmission and Distribution, 152(6), 899-905.

[13] Venkaiah Ch.; Vinod Kumar D. M. (2011). Fuzzy PSO Congestion Management using Sensitivity-Based Optimal Active Power Rescheduling of Generators. Journal of Electrical Engineering \& Technology, 6(1):32-41.

[14] Ghasemi A., Khalil V., Akbar T. (2014). Multi objective optimal reactive power dispatch using a new multi objective strategy. Electr Power Energy Syst, 57:318-334. 